\title{
Delboeuf “illusions” of circle size induced via photoflash-generated afterimages
}

\author{
WILLIAM J. O'HALLORAN \\ LeMoyne College, Syracuse, New York 13214 \\ and \\ DANIEL J. WEINTRAUB \\ University of Michigan, Ann Arbor, Michigan 48109
}

\begin{abstract}
Generating an inducing figure by photoflash led to underestimation of the size of a luminous outline test circle in a nonluminous surround. As the afterimage of the inducing circle decayed, judged size of the test circle attained true size. The more intense inducing flash produced a longer lasting perceptible afterimage but no alteration in amount of test-circle underestimation as a function of time. The type of inducing configuration (outer concentric circle, nonconcentric circles, arcs) proved to have no differential effect. In the absence of an inducing figure, subjects make veridical judgments.
\end{abstract}

The Delboeuf display, hereafter to be called the concentric Delboeuf in order to distinguish it from several variations, consists of a pair of concentric circles. The circle whose size is to be judged is called the test circle. The circle (or other contour) that is presumed to cause the anomaly (illusion) is called the inducing stimulus. Separated laterally from the test-inducing combination is a single circle, the comparison, against which the size of the test circle is judged. Two other Delboeuf variations were employed (see the illustrations in Figure 1): the nonconcentric-Delboeuf display (Titchener's circles, Ebbinghaus's figure) consisting of test circle flanked by an inducing configuration of four larger circles, and the arc-Delboeuf display, in which the inducing stimulus was a truncated version of the concentric inducing stimulus, the $25 \%$ of each circumference closest to the test circle. All elements of the displaytest, inducing, and comparison-can be presented simultaneously, the normal mode for viewing these geometrical visual anomalies. However, the inducing configurations can be presented first, following by the test-comparison circles, the so-called figural aftereffects paradigm.

Theorists have sought an explanation from two basically different starting points. The functionalist

We thank Mr. Robert M. Barrett for his work in Experiment 2. The investigation was supported in part by a United States Public Health Service Research Scientist Development Award (K2-MH-35253) to the second author from the National Institute of Mental Health. Research funds were provided partly by National Science Foundation Grant GB 8181 and partly by Grant MH 23767 from the National Institute of Mental Health. Requests for reprints should be sent to William J. O'Halloran, LeMoyne College, LeMoyne Heights, Syracuse, New York 13214. approach appeals to cognitive factors, comparisons of sizes or distances between display elements leading to misjudgments of circle size. The contourdisplacement approach appeals to mechanisms of interaction between the neural representations of adjacent display elements. (See Cooper \& Weintraub, 1970 , for an extended treatment, including developmental implications.) At least two factors are relevant, the cognitive or noncognitive nature of the phenomena and the neural mechanisms involved (including their loci). A point of experimental attack with important theoretical implications is the introduction of a successive-presentations methodology in which the inducing figure is presented by means of a photoflash unit so that a perceptible, long-lasting afterimage is produced. The test stimulus can be presented periodically to assess the magnitude of size misjudgments as time passes. The usual procedure is to require the observer to fixate the inducing stimulus for a matter of seconds or minutes. The photoflash method, however, reduces retinal smearing of the afterimage because of inability to fixate steadily, eye tremor, etc. Another methodological improvement is the use of luminous outline stimuli in black (zero luminance) surrounds. A possible confounding variable, the contour formed at the border of a display, is eliminated so that only the contours of inducing, test, and comparison stimuli, plus an ever present fixation point, are visible.

A neural theory of size misjudgments must account for the fact that a more intense flash of the inducing stimulus produces a more intense, longer lasting afterimage (see Ganz, 1966a, pp. 154-156). The neural trace can be expected to mirror the anomaly. Thus, a photochemically or neurally based 
theory would seem to require that the brighter the flash causing the afterimage, the more pronounced will the anomaly be initially, or the longer lasting, or both. Second, the time course of the disappearance of the anomaly might be expected to be correlated positively with the reported disappearance of the afterimage when flash luminance is varied.

Massaro and Anderson (1971) have presented evidence that the nonconcentric Delboeuf display under simultaneous-presentation conditions gives data compatible with the hypothesis that observers are comparing the sizes of test and inducing figures. Girgus, Coren, and Agdern (1972) and Weintraub and Cooper (1972) concur, but argue that contourinteraction factors are also involved. An identical explanation for the concentric Delboeuf seems unlikely. The concentric Delboeuf leads to "assimilation," the inner circle appearing too large with simultaneous conditions, but leads to "contrast," the inner circle appearing too small with successivepresentation conditions. However, when inducing circles are considerably larger than the test circle, the nonconcentric Delboeuf is always a "contrast" anomaly (Cooper \& Weintraub, 1970, moderate-tolow-intensity inducing figures and nonluminous surrounds). If a perceptible afterimage of the inducing figure is produced by means of a bright flash, will the concentric-Delboeuf display become an "assimilation" anomaly under these successivepresentation conditions? In other words, is perceptibility the crucial variable for psychological simultaneity? Afterimage perceptibility may or may not facilitate cognitive size comparisons, because perceptibility could be irrelevant if only memory for size is important. Ganz (1966a, 1966b) postulated that simultaneous and successive effects were aspects of the same underlying neural processes. What persists over time in the successive case, to act upon the test figure, is the trace of the inducing figure. One proof of the existence of a trace is the presence of a perceptible afterimage, so the use of perceptible afterimages is a technique that may help unravel the apparent contradictions. The arc-Delboeuf display contains close-in contours identical to those of the nonconcentric Delboeuf, but reduces the sizecomparison possibilities inherent in the concentric and nonconcentric displays.

The research strategy involved mainpulating two variables with theoretical significance: the type of inducing configuration and the intensity of the inducing flash.

\section{EXPERIMENT 1}

\section{Method}

Subjects. The 48 subjects were volunteers, students or staff, for the most part, at the University of Michigan and the College of the Holy Cross.
Apparatus. The light-tight viewing tunnel contained a headrest at one end and a holder for stimulus materials at the other. A box containing three 4-W daylight fluorescent tubes behind a translucent Plexiglas panel provided diffuse backlighting for a comparison circle and a test circle inserted in the stimulus holder. Luminance of each outline circle was $34.3 \mathrm{~cd} / \mathrm{m}^{2}$. On the side of the tunnel, near the end-mounted holder, was another stimulus holder coupled to a photoflash unit. The photoflash was a Honeywell (64B) electronic strobe (manufacturer's specifications: $50 \mathrm{~lm} \mathrm{sec} / \mathrm{ft}^{2}$ at $\left.4 \mathrm{ft}\right)$ covered by flashed opalglass $(52 \%$ transmission) for even illumination. The photoflash backlighted the inducing configuration placed in the side holder. The image proceeded to the subject's eyes via a front-surface mirror in a hinged mount set at a 45-deg angle in the tunnel. From the subject's point of view, all stimulus elements, test, comparison, and inducing, appeared in the dark as luminous outline stimuli in a plane normal to the line of sight, $1.25 \mathrm{~m}$ from his eyes. A dim fixation light, centered between comparison and test circles was always visible. (The mirror contained a horizontal slot so that the fixation light could be seen by both eyes even with the mirror in the line of sight.)

Retouched high-contrast photographic negatives were used as stimuli. The test figure, $20.0 \mathrm{~mm}$ in diameter, was presented to the right of the fixation light. The diameter of the inducing circle of the concentric Delboeuf was $30 \mathrm{~mm}$. The four inducing circles of the nonconcentric Delboeuf were $40 \mathrm{~mm}$ in diameter. The distance between the center of each nonconcentric inducing circle and the center of the test circle would have been $34.0 \mathrm{~mm}$ if inducing and test elements had actually been in the same plane. The four inducing arcs of the arc Delboeuf consisted of the innermost $25 \%$ of the circumference of the nonconcentric inducing circles. If inducing and test figures had been presented simultaneously, they would have appeared to the subject as in the illustrations of Figure 1. One of a set of comparison circles, which differed by $.5-\mathrm{mm}$ steps, was presented to the left of the fixation point. The line thickness of all figures was $.4 \mathrm{~mm}$; the horizontal distance between the centers of comparison and test circles was $76 \mathrm{~mm}$.

Procedure. The subject was instructed to fixate the fixation point whenever stimuli were being presented. A session proceeded as follows: After dark adapting for $3 \mathrm{~min}$, the subject fixated the luminous point in an otherwise dark field, and triggered the photoflash himself. The bright flash left a perceptible afterimage of the inducing figure. The experimenter immediately swung the front-surface mirror out of the subject's line of sight so that the test and comparison circles could be simultaneously presented for the first size judgment. Following the flash, test and comparison circles appeared periodically throughout an 8 -min period, first at $1.5 \mathrm{sec}$, then at every $1 / 2 \mathrm{~min}$ following the flash to the 2 -min mark, then every minute thereafter. The subject was required to fixate the fixation point during the 1.5 -sec interval when the test-comparison pair appeared for each judgment. In addition to size judgments, the subject was continually asked whether the afterimage of the inducing stimulus was still visible, and the reported time of disappearance was recorded. To minimize carryover of afterimage effects, each session consisted of only one flash, with a minimum delay of $3 \mathrm{~h}$ imposed between sessions. There were 9 sessions per subject. In random order, the three inducing configurations, concentric, nonconcentric, arc, were each presented at three luminance levels: full flash intensity, flash reduced by 1.0 neutral-density filter ( $10 \%$ transmission), flash reduced by 2.0 filter ( $1 \%$ transmission). Kodak Wratten neutral-density filters were used. Control data, test circle judged against comparison in the absence of an inducing flash, were taken at the beginning of the first session and at the beginning of the last session. A variant of the method of constant stimuli was employed, the group version of the up-and-down (staircase) technique (Hanley \& Zerbolio, 1965). In accordance with the method, whenever a test circle was presented for judgment, it was paired with a comparison circle whose size was pre- 
determined by the previous subject's response to that specific combination of inducing configuration, inducing luminance, and time delay between inducing and test configurations. For the first subject, the experimenter selected randomly a comparison circle, not differing by more than $1.0 \mathrm{~mm}$ from the test circle, to be paired with each test circle. The subject was required to state whether the comparison circle was larger or smaller than the test circle. Each subject contributed only one judgment toward data needed to compute each mean. As the data accumulate across subjects, the responses tend to oscillate about the point of subjective equality for size. Separate up-and-down sequences, 99 in all, were maintained, one for each of the 9 conditions at each of 11 time intervals. The method of data analysis, which differs somewhat from the conventional up-and-down statistical treatment (Dixon \& Massey, 1957), is described by Weintraub, Wilson, Greene, and Palmquist (1969).

\section{Results}

The variable of interest is the judged size of the test circle as a function of elapsed time since the inducing flash. Each datum constitutes the mean diameter of the comparison stimulus judged equal to the test stimulus under the given conditions. Mean values smaller than $20.0 \mathrm{~mm}$ indicate a "contrast" (diminution) effect. There were essentially no differences among the 9 conditions ( 3 filter densities $\times 3$ configurations) as a function of elapsed time. Main effects are plotted in Figure 1, the effect of filter density averaged across inducing-figure configurations, and of inducing-figure configurations averaged across filter densities. For the means displayed in Figure 1 at an elapsed time of nearly zero, standard errors of the mean had an average value of $.13 \mathrm{~mm}$ (maximum $\mathrm{s}_{\overline{\mathrm{x}}}=.17 \mathrm{~mm}$ ). A $99.99 \%$ confidence interval about any of these means would never include the null result (i.e., would not include a judged diameter of $20 \mathrm{~mm}$ ). At an clapsed time of $1 / 2 \mathrm{~min}$, a $95 \%$ confidence interval about each mean would not include the null result in three of the six instances (average $s_{\bar{x}}=.18 \mathrm{~mm}$ ), and, for elapsed times thereafter, one would have little confidence that size underestimation had occurred. (To characterize the approach to the steady state, for elapsed times of $1,11 / 2,2,3$, and $4 \mathrm{~min}$, average $s_{\bar{x}}$ values were $.17, .18, .17, .17$, and $.16 \mathrm{~mm}$, respectively.) We are not trying to argue that confidence intervals provide the criterion for deciding when an effect has dissipated, but merely to demonstrate that, since hefty confidence intervals do not cover the null outcome for early size misestimations, it is a very safe bet that these underestimates are genuine. The result is clearcut. The afterimage technique produced a .8-mm size underestimation that disappeared in a matter of minutes. The effect, though striking, was independent of the type of inducing figure and of flash intensity. During the course of a session, subjects were also asked to judge whether the afterimage was still perceptible. The mean times of reported disappearance for filter densities 0,1 , and 2 were $64.6,60.6$, and $53.2 \mathrm{sec}$, respectively $[F(2,48)=$

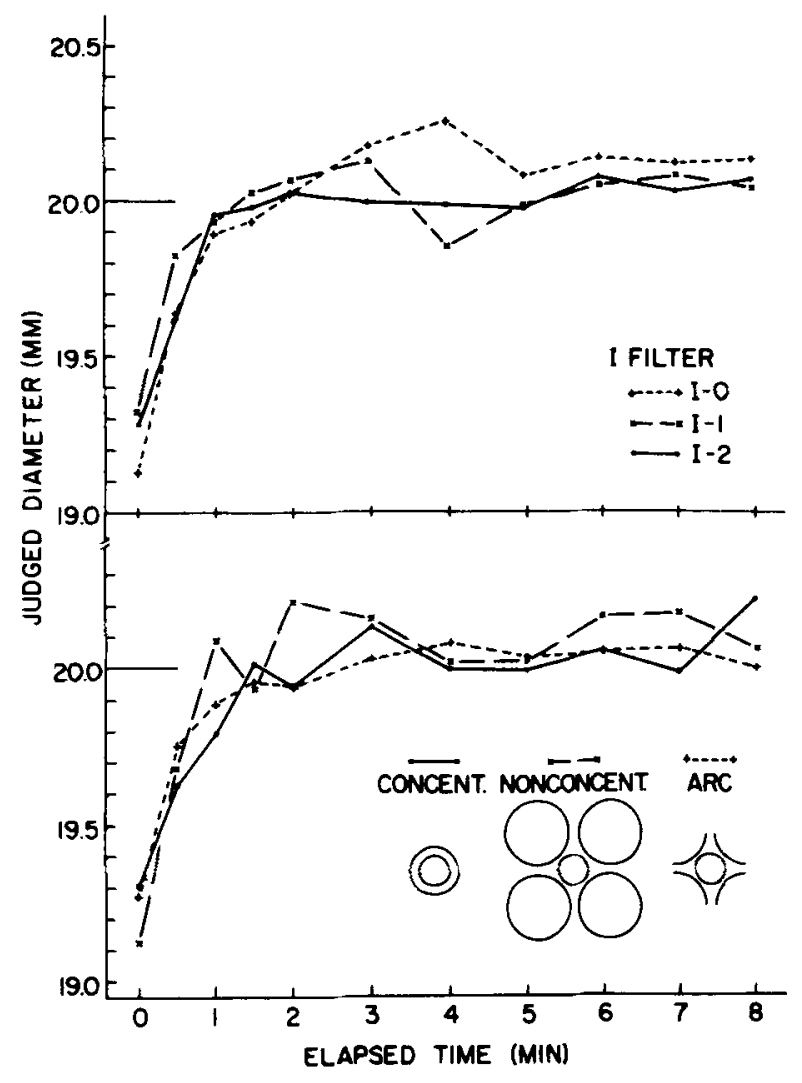

Figure 1. Data from Experiment 1 showing the judged size of lest circle following an inducing-figure afterimage at each of three levels of stimulus intensity (upper half) and for each of three Delboeuf displays (lower half). (True diameter equals $20.0 \mathrm{~mm}$.)

5.91, significant at $\propto=.01]$. Thus, the brighter the flash, the longer lasting was the afterimage. The analysis of variance also showed no significant main effect of the type of inducing configuration, and no interaction between filter density and configuration. It should be noted that the presence or absence of a weak afterimage is not easy to determine. Only 17 subjects managed to provide a judgment for every session. A highly conservative analysis of variance procedure was followed, eliminating 8 subjects who provided fewer than seven of the nine judgments, and reducing the df of every F-ratio denominator by the number of data points that were estimated.

In sum, an inducing figure presented as an afterimage effected a change in the judged size of the test figure, and this change, an underestimation, disappeared over time. While the duration of the afterimage was linked to flash intensity, the data for size misjudgments showed no discernible differential effect of it.

\section{Discussion}

There is some indication that anomalies of judged size outlast the reported duration of afterimages, 
which is very likely to be true. (See a discussion by Hochberg, 1971.) The severe criterion problem in deciding whether an afterimage is no longer visible makes difficult the comparison of the reported duration of afterimages with the duration of size effects. An important and defensible conclusion is that flash intensities over a range of $2 \log$ units produced a potent size anomaly that dissipated with time, but no detectable differential effects, as the upper half of Figure 1 attests. Ikeda and Obonai (1953) found that, when the independent variable was the length of time subjects fixated the inducing figure, rather than its intensity, the initial magnitude of the effect was not altered, but it diminished more slowly for long fixations.

Simultaneous presentation of all elements of a Delboeuf display has shown that a larger concentric inducing circle leads to size overestimation ("assimilation"), larger nonconcentric circles to underestimation ("contrast"), with arc data lying somewhere between (Cooper \& Weintraub, 1970). The finding that these inducing configurations, presented as perceptible afterimages, produced identical underestimation effects is especially important, because it demonstrates that a perceptible afterimage of the inducing stimulus does not act like the retinal image of the physically present inducing stimulus.

An incidental finding was that a control trial, comparison against test circle in the absence of an inducing circle, at the beginning of the initial session provided a mean judged size of $19.65 \mathrm{~mm}$, while the same control just prior to the last session provided a mean of 20.29 . Although the rise of $.64 \mathrm{~mm}$ in judged size does not quite achieve statistical significance (.05 level $\mathrm{t}$ test for nonindependent samples), these data hint that a trend of increasing size judgments may occur across testing sessions, a matter of days, even though the underestimation effects of any flash seem to have disappeared completely within $3 \mathrm{~min}$.

\section{EXPERIMENT 2}

The control trials of Experiment 1 had been run at the onset of the first experimental session and again just prior to the last experimental session. To furnish additional certainty, it was decided to run an entire experiment of control trials and to have the subjects judge, over a 5-min period, the size of a 20 -mm luminous test circle in a nonluminous surround in the absence of any inducing figure.

\footnotetext{
Method

Subjects. The 54 subjects were new volunteers, primarily students at the College of the Holy Cross.

Apparatus. The apparatus was the same as that used in Experiment 1 . The flash attachment was not used.
}

Procedure. With the exception of the steps needed to induce the afterimage, the procedure was the same as that of Experiment 1. The subject, dark adapted and fixating, judged the size of the 20-mm-diam test circle over a 5 -min period at 30 -sec intervals. Comparison circles were presented and means computed as in Experiment 1.

\section{Results}

The 11 "control" judgments produced an essentially horizontal function. The mean at each time interval was: $20.02,20.19,19.91,20.00,20.18$, $19.97,20.10,19.94,19.96,20.06$, and 20.13. The overall mean of the series is 20.04 , with $.19 \mathrm{~mm}$ as the largest deviation from $20 \mathrm{~mm}$.

\section{Discussion}

The results of Experiment 2 confirm the validity of the data of Experiment 1 in showing clearly that in the absence of any inducing configuration, subjects judge a $20-\mathrm{mm}$ test circle veridically. Therefore, the initial size underestimation in Experiment 1 depends on the presentation of an inducing figure and is not the result of any sequential effects that might occur within a series of judgments taken $30 \mathrm{sec}$ apart.

\section{CONCLUSIONS}

What do the data imply about the Delboeuf effect? In previous studies (Cooper \& Weintraub, 1970; Weintraub \& Cooper, 1972) using the identical three inducing configurations employed in Experiment 1, when the inducing and test figures were presented simultaneously, substantial differences in the magnitude and direction of the effects were obtained. If perceptibility is the crucial variable for psychological simultaneity, then the perceptible afterimage produced by a high-intensity inducing flash might alter the size of the test circle in the same manner as an inducing stimulus that is physically present. The perceived afterimage of a larger concentric inducing circle would then act "cognitively" like the perceived image of an objectively present inducing circle, producing size overestimation. This did not occur. Interestingly, even though the concentricDelboeuf effect was reversed, the afterimage procedure did not lead to reversed size judgments for nonconcentric and arc configurations. Taken in the aggregate, the data show that the different inducing configurations act equivalently only when the afterimage procedure is used. Therefore, in influencing the judged size of the circles, a noticeably perceptible afterimage does not act like a physically present stimulus. Thus, the new evidence does not permit a simple extension of cognitively based theories to the afterimage-induced Delboeuf effect. However, area, length, or distance comparisons within the stimulus field may be unusual, difficult, or perhaps 
impossible when the inducing "stimulus" is a not-socrisply defined afterimage fixed upon the retina while the test stimulus is the usual unfixed, clear, bright, retinal representation. It is not possible, therefore, to rule out cognitive theories on the basis of these nonsupportive data. (Afterimage data matching those for simultaneously presented inducing and test figures would have been very diagnostic.)

The evidence contradicts the theory that an afterimage forges the link between simultaneous and successive modes of presentation (Ganz, 1966a, 1966b). What has been assumed to be bridging the time gap in a figural-aftereffects procedure is the afterimage of the inducing figure. If that be literally true, then there should certainly be size overestimation for the concentric configuration in the present experiment. There is not. In addition, both afterimages and size misjudgments should be dependent upon the absolute intensity of stimulation. Afterimages are, but size misjudgments are not detectably so.

The evidence concerning the ineffectiveness of inducing-figure luminosity differences (other than the control condition, which can be considered as zero luminance) can be placed in the perspective of previous evidence. Luminance contrast, the ratio of figure luminance to surround luminance (or the inverse if the surround is more luminous), has proved to be a crucial variable. With nonzero background luminances for the inducing figure, Graham (1961) found, in a figural-aftereffects line-displacement experiment, that increasing the luminance contrast of the inducing figure increased the judgmental error. When luminance contrast was held constant and the entire display containing the inducing figure was intensified throughout a range of nearly $5 \mathrm{log}$ units, there was no significant change in error magnitude. With the simultaneous concentricDelboeuf display presented as dark stimuli on light surrounds, increasing the luminance contrast of the inducing figure increased the error magnitude (Oyama, 1962; Weintraub \& Cooper, 1972; Weintraub et al., 1969). An experiment by Cooper and Weintraub (1970) found luminance to be irrelevant for Delboeuftype inducing stimuli of moderate luminance (4 to $\left.111 \mathrm{~cd} / \mathrm{m}^{2}\right)$ in nonluminous surrounds. The evidence is consistent with the hypothesis that the important luminosity variable pertaining to inducing figures is not absolute luminosity, but rather luminance contrast. In eliminating all extraneous contours (except the fixation point) in the present experiment, background luminance was eliminated. Thus, all inducing-flash intensities approached infinite luminance contrast, and therefore, being equal in luminance contrast, were equally effective.

Let us close with a bit of informed speculation based upon the following evidence: First, luminance contrast crucially affects the potency of inducing figures as discussed in the previous paragraph. Second, luminance contrast also influences the susceptibility of test figures embedded in luminous surrounds (Weintraub \& Cooper, 1972). Third, a size/luminance-contrast anomaly exists such that reducing the luminance contrast of a test figure reduces its judged size in the absence of any inducing component (as demonstrated by Weintraub, Tong, and Smith, 1973, using vertical lines as stimuli). On the basis of such luminance-contrast findings plus the afterimage-Delboeuf results, we propose that the neural contour associated with an outer inducing figure (of a given luminance contrast) acts on the test circle to reduce its effective luminance contrast (the neural representation of luminance contrast) by adding effective luminance (the neural representation of luminance underlying "perceived luminance," i.e., brightness) to the surround in the manner that a flash bulb leaves a fading afterglow in an otherwise dark visual field. And so, the hypothesis is that size misjudgments are actually the result of the size/ luminance-contrast anomaly, which diminishes the judged size of the test circle by increasing the effective luminance of the surround.

\section{REFERENCES}

Cooper, L. A., \& Weintraub, D. J. Delboeuf-type circle illusions: Interactions among luminance, temporal characteristics, and inducing-figure variations. Journal of Experimental Psychology, $1970,85,75-82$.

Dixon, W. J., \& MAsseY, F. J. Introduction to statistical analysis (2nd ed.). New York: McGraw-Hill, 1957.

GANZ, L. Is the figural aftereffect an aftereffect? A review of its intensity, onset, decay, and transfer characteristics. Psychological Bulletin, 1966, 66, 151-165. (a)

Ganz, L. Mechanism of the figural aftereffect. Psychological Review, 1966, 73, 128-150. (b)

Girgus, J. S., Coren, S., \& Agdern. M. The interrelationship between the Ebbinghaus and Delboeuf illusions. Journal of Experimental Psychology, 1972, 95, 453-455.

Graham, E. H. Figural after-effects as functions of contrast, area, and luminance of the inspection-figure. Psychologia, 1961, 4. 201-208.

Hanley, C., \& Zerbolio. D. J. Developmental changes in five illusions measured by the up-and-down method. Child Development, 1965, 36, 437-452.

Hochberg, J. Perception: Color and shape. In J. W. Kling and L. A. Riggs (Eds.), Woodworth \& Schlosberg's experimental psychology. New York: Holt, Rinehart, \& Winston, 1971.

IKEDA, H., \& ObonaI, T. The quantitative analysis of figural aftereffect (I). The process of growth and decay of figural aftereffect. Japanese Journal of Psychology, 1953, 24, 59-68.

Massaro, D. W., \& Anderson, N. H. Judgmental model of the Ebbinghaus illusion. Journal of Experimental Psychology, 1971. 89, 147-151.

Oy AMA, $T$. The effect of hue and brightness on the size-illusion of concentric circles. American Journal of Psychology, 1962, 75, 45-55.

Weintraub, D. J., \& Cooper, L. A. Coming of age with the Delboeuf illusion: Brightness contrast, congition, and perceptual development. Developmental Psychology, 1972, 6, 187-197.

Weintraub, D. J., Tong, L., \& Smith, A. J. Müller-Lyer versus 
size/reflentance-contrast illusion: Is the age-related decrement caused by a declining sensitivity to brightness contours? Developmental Psychology, 1973, 8, 6-15.

Weintraub, D. J.. Wilson, B. A., Greene, R. D., \& Palmoutst. M. J. Delboeuf illusion: Displacement versus diame- ter. arc delections. and brightness contrast. Journal of Experimental Psychologi'. 1969, 80, 505-511.

(Received for publication May, 1977; accepted May 9, 1977.) 